\title{
Alternative models and methods to animal experiments in vascular diseases
}

\author{
F Passariello* \\ *Fondazione Vasculab ONLUS, via Francesco Cilea 280 - 80127 Naples, Italy
}

presented to: the Hemodyn2015, Nov 2015, Naples, Italy submitted: Nov 5, 2016, accepted: Nov 21, 2016, EPub Ahead of Print: Nov 30, 2016, published: Dec 31, 2016 Conflict of interest: None

Corresponding author: Dr. Fausto Passariello, afunzionale@tiscalinet.it

(C) 2016 Fondazione Vasculab impresa sociale ONLUS. All rights reserved.

Abstract The Vasculab Foundation aims to find alternative models to animal experiments. Even thinking that animal experiments are useful, it is necessary to respect the 3Rs rule: Replacement, Reduction, Refinement If the 3Rs strategy would be used in the daily experimental work, a strong reduction in the use of animals in laboratories would be observed. Only a few examples are reported here, in particular those referring to the vascular contest, although the argument is much wider in other fields of biomedical research. Other fundamental issues regard the real innovation and the conflict of interest unacceptable in human trials but accepted in animal research. Finally, a good researcher should be the one who defends the animals used in the experiments, taking the part of the animals, instead of gaining from their use only in the name of an untouchable research interest.

Keywords Animal rights, non-animal experiments, the 3Rs argument, replacement, reduction, refinement

\section{Introduction}

One of the proposals of the Vasculab Foundation is to find alternative models to animal experiments and this is a proof of love and respect on the part of the Foundation towards animal life. However, these reasons could be considered not sufficient to all scientists to find alternative methods to animal experiments. The first thing we need to ask about is

"whom is this message addressed to?".
What do I want to speak about? Do I want to speak about ethical issues? Well, I am in favour of talking about ethical issues, but I will not do that; or do I want to speak of the usefulness/uselessness of animal experiments? I could do that, but I don't want to do it, because I want to talk of a completely different topic:

Even if you think that animal experiments are useful, you must respect the rules

This is a practical topic addressed to researchers, it is not a struggle nor an ideal crusade.

What are the rules?

There are a lot of declarations, institutional declarations $^{1}$, associations ${ }^{2}$ and scientific societies like the European Society for Alternatives to Animal Testing $(\text { EUSAAT })^{3}$, which are involved in explaining these rules.

Let me report just an example of experiments which do not follow the rules. Generally, many people are not aware that scientists used for a long time several useless animal toxicological tests to study cosmetic drugs and home care detergents, while they could have simply used practical non-animal tests that were already available. Underestimating these facts has no ethical justification at all.

But even if you are convinced that the animal experiments are absolutely useful, you must respect the 3Rs argument: Replacement, Reduction, Refinement. (Figure 1) Then there are other fundamental questions ... and last but not least several final remarks. 


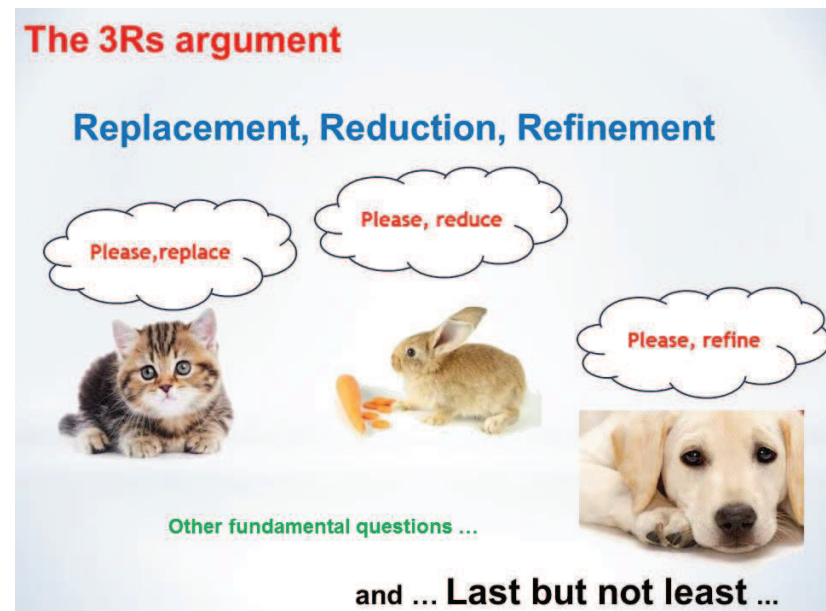

Figure 1 - The 3Rs argument

\section{Replacement}

Please, replace (if you can)

your animal experiment with another (almost equivalent) experimental set, which does not require at all the use of any animal. As examples, we will illustrate only experiments of interest in vascular research.

\section{Physical experimental sets}

A classic replacement in hemodynamic measurements (pressure, flow, etc.) is provided by a Mariotte bottle ${ }^{4}$ (Figure 2), used to fix the input pressure, and a Penrose drainage tube, which simulates a collapsible vessel, together with a hydraulic or an electrical manometer ${ }^{5,6}$ (Figure 3). This experimental set is easily adapted to several research problems, involving also the effect of the tissue pressure on a collapsible vessel, easily simulated by a jacket with a second manometer. It is possible to change fluids input and calibres, resistance, height of the containers, and then take several measurements on flow, input and output pressures and so on, using a very low cost non-animal set.

The experiments can be repeated as many times as we want, provided we do not change the experimental set. For instance, injecting a glue inside the Penrose drain, alters the device and in order to set-up the subsequent experiment it is necessary to change the drain and any other altered component (Figure 3).

At the exit of the Mariotte bottle, the input pressure to the device is given by the difference in height between the level of the input tube in the bottle and the level of the experimental tube. As long as the liquid level is maintained higher than the extreme of the input tube, the input pressure remains constant.

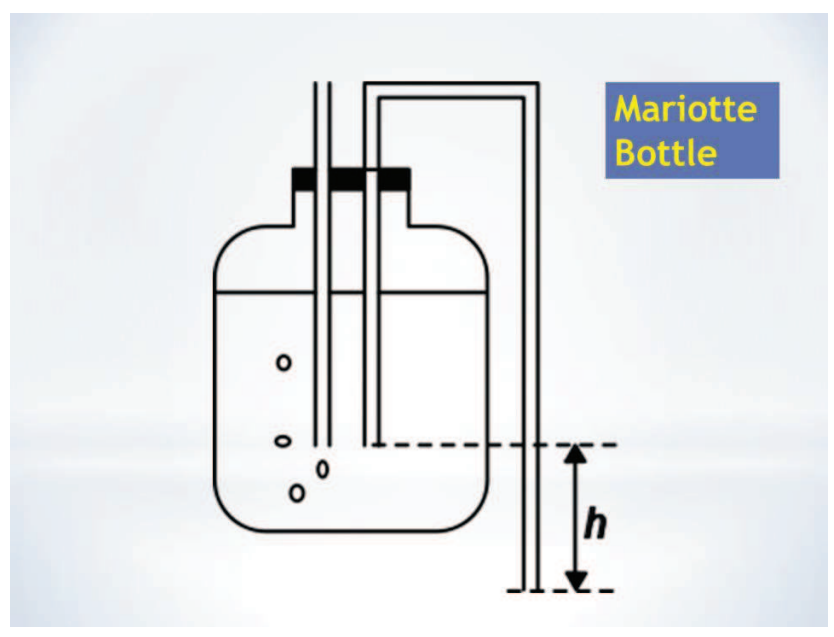

Figure 2 - Mariotte Bottle

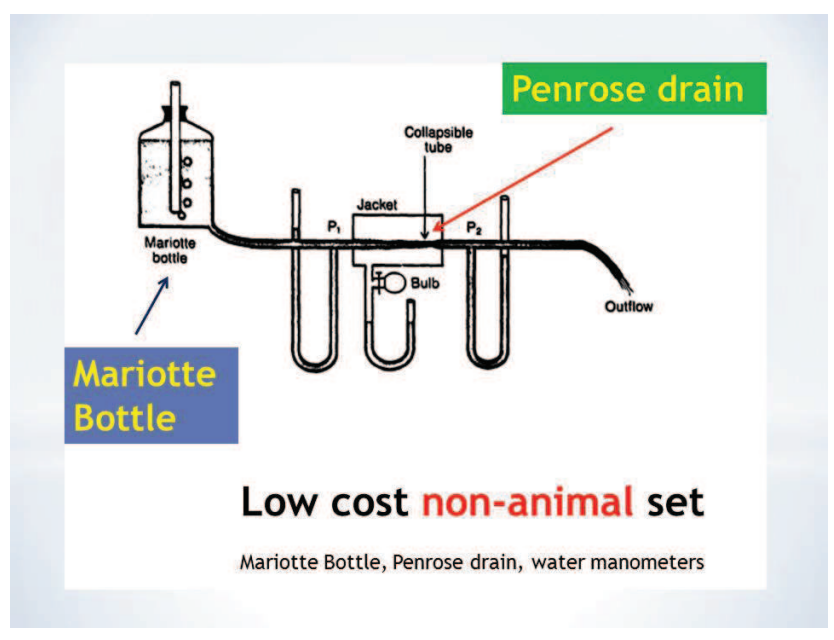

Figure 3 - Low cost non-animal set

Penrose drains are generally used to promote drainage in open surgical wounds and are easily found on the market. They are available in flat shape and several sizes (12-36 in) and widths (1/4-1 in), as in sterile as well non-sterile packages (Figure 4).

\section{Computer simulations}

It is possible to estimate pressures and tensions (wall and venous valves) in a simplified clinical setting in the saphenous-femoral junction. Though the hidden complexity of computation, which however is faced only once and embedded in an opportune algorithm, measures are very simple and results can be very satisfying ${ }^{7}$.

A lot of useful simulations can be organized, in order to understand better the experimental problem and to get help in designing the right experiment (see the "Refine" paragraph). Let me cite just one example constituted by 
computer simulations made using Computational Fluid Dynamics programs ${ }^{8}$, which can simulate very complicate models in hemodynamics.

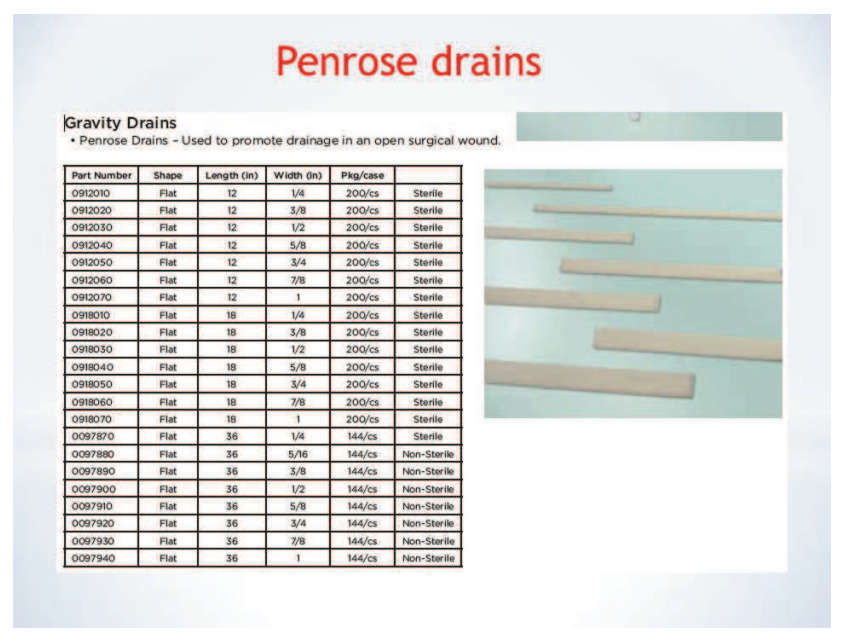

Figure 4 - Penrose drains

\section{Non-animal ultrasound pads and phantoms}

Often a slice of turkey meat is used as a low cost ultrasound pad in emergency vascular access courses, while animal pads are not used for LASER endovascular treatments and venous sclerotherapy.

However, it is possible to build simply and quickly low cost pads for training in emergency and vascular access courses. Although the majority of these methods adopts homemade gelatine based ultrasound pads ${ }^{9-11}$, it is possible to build homemade non-animal agarose ultrasound pads ${ }^{12}$, including objects and Penrose drains to simulate tissue structures and vascular vessels for training in vascular access. Another alternative is given by high cost pads distributed by ultrasound companies, which offer a much greater reliability.

Replacing in favour of non-animal experiments can sometimes result on a reduction of costs and other times on an increase in costs, as these economic features depend mainly on the specific experimental set and design, thus a general answer cannot be provided.

\section{Reduction}

\section{Please, reduce (if you can)}

the number of animals required to perform your experiment. Benefit of statistics to compute the power of your experimental design ${ }^{13}$.

What does it mean?

\section{Example of reduction}

Given $\mathrm{N}$ the number of required measures to get the optimal power in your experimental design and $M$ the number of the measures you planned, consider that:

- using $\mathbf{M}>\mathbf{N}$ measures, you will waste animal life and/or provide useless animal pain.

- using $\mathbf{M}<\mathbf{N}$, again you will waste animal life and/or provide useless animal pain, because your experiment will have no power and no meaning at all and the sacrifice of all the animal used in the $\mathrm{M}$ measures will be absolutely vane.

- using $\mathbf{M}=\mathbf{N}$ measures, or a little bit more (because some measures could be lost), you will do the right job, performing a reliable research with the right number of animals, therefore respecting the animal life.

Computing the right number $\mathrm{N}$ is not difficult, but requires a little knowledge of statistical methods ${ }^{13}$. This topic will be treated in depth later in a future article.

In addition, reducing has also an important effect in decreasing the overall cost of the experiment, decreasing the number of animals and the amount of materials used in each repetition, as well as the effort of people involved in the experimental work.

\section{Refinement}

\section{Please, refine (if you can)}

your experimental design and your underlying theory. Maybe, making computations and ameliorating your experimental planning, dedicating some effort to perfecting the design multiplying by 2 or 3 the time you already allotted to this job (NB! to be done only once), you will get a more meaningful experiment with better results, i.e. you will improve the experiment reliability.

Refining, can get a reduction in costs, when a simplification of the experimental set is achieved. Generally, a well done experiment can only get advantages in resources allocation from refining, while a badly conceived experiment can even require an increase in complexity and hence in costs. However, we should agree that a badly conceived experiment should never be carried on, being scientifically useless and in addition nonrespectful of the animal rights and lives.

Really, the abundance of non-well-conducted animal experiments and the almost absence of systematic reviews and meta-analyses in animal research was recently pointed out in an important medical journal ${ }^{14}$. When translated later to human research these bias expose human participants to clinical trials to several harmful drug effects. Such a wastage of human and animal resources "is as unethical in animal as in human research"14.

Finally, an important "refinement" regards the animal sacrifice after animal experiments. Do you really need this 
sacrifice? For instance, you need to sacrifice an animal if you must get data from a lethal extensive tissue sampling.

On the contrary, could you help the animal in restoring its previous health conditions?

\section{Other fundamental questions}

Even if you will not communicate to other people the answers, nevertheless you have to stand in front at the mirror and ask yourself the following questions:

- Are you sure that you are making an innovative experiment which had not been already done before? Are you repeating instead an already published research? Repeating a research can still have a meaning, if you deliberately repeat a previous research of literature to check its reliability, but not if you are not aware of it in previous experiments. Knowledge is a prerequisite of good research, not being aware of literature is only ignorance.

- Which are the true motivations to perform your investigation? To which extent personal or team interests are involved? In simple words, can you recognize in your experiment anything, maybe an economic involvement, which in human experiments could be easily called a "conflict of interest"? And, if this is the case, which justifications do you have to underestimate this conflict of interest while performing the animal experiment, while you feel obliged to take it into account while doing human research?

As an example, let's consider an experiment on the effect of a new drug, which is produced by a company. Having assets and personal interests in the company is generally considered a conflict of interest in human research, because an even unconscious bias, could be present in the results, vanishing then the usefulness of the human research. Why this identical argument does not stand for animal experiments? The same conflict would frustrate also the usefulness of the animal research.

The only explanation is that there is no Ethical Committee checking on the experimental design in almost the totality of animal research nor any Scientific Committee to evaluate the real usefulness of animal experiments.

\section{Last but not least...}

Consider that nothing (except some very weak laws) defends the animals.

As you are the planner and the owner of your experiment, you must take the part of the animals. Nowadays no one maybe will control you, but remind yourself that you are the only one who can defend the animals you want to use in your experiments, you are like a supervisor, you are the one who owns their lives.

Do not betray them!

\section{References}

1) Bankowski Z, Howard-Jones N. International Guiding Principles for Biomedical Research Involving Animals. The Council for International Organizations of Medical Sciences (CIOMS), 1985. Available at the address http://www.cioms.ch/index.php/publications/ available-publications/540/view_bl/61/bioethics-andhealth-policy/20/international-guiding-principles-forbiomedical-research-involving-animals, at the date of Nov 7th, 2016.

2) The Alternatives web site. Available at the address https://thealternativeseu.wordpress.com, at the date of Nov 6th, 2016.

3) European society for alternatives to animal testing. Available at the address http://www.eusaat-congress.eu/, at the date of Nov 7th, 2016.

4) Holt, JP. Flow through collapsible tubes and through in situ veins. IEEE T. Biomed. Eng. 1969; 16:274-283.

5) Permutt S, Riley RL. Hemodynamics of collapsible vessels with tone: The vascular waterfall. J Appl Physiol 18:924-932.1963.

6) Raju S, Varney E, Flowers W, Cruse G..Effect of External Positive and Negative Pressure on Venous Flow in an Experimental Model. Eur J Vasc Endovasc Surg 2016; 51(2):275-284.

7) Passariello F. Estimation of pressure and flow in the saphenous femoral junction. Presentation to the Hemodyn2015 Meeting of the Vasculab Foundation, Napoli, Nov 3-5, 2015. Available at the address http://www.vasculab.it/hemodyn2015/participants.htm at the date of Nov 7th, 2016.

8) Munson BR, Young DF, Okiishi TH. Fundamentals of Fluid Mechanics. Fifth Edition. John Wiley \& Sons, Inc. 2006.

9) Bude RO, Adler RS. An Easily Made, Low-Cost, Tissue-Like Ultrasound Phantom Material. J Clin Ultrasound 1995;23(4):271-273. https:// deepblue.lib.umich.edu/bitstream/

handle/2027.42/38198/1870230413 ftp.pdf?sequence=1, at the date of Nov 7th, 2016.

10) Emme S. Homemade ultrasound iv model. https:// www.youtube.com/watch? $=y p w 8 v j Z 2 D N 0$, at the date of Nov 7th, 2016.

11) Cheruparambath V, Sampath S, Deshikar LN, Ismail HM, Bhuvana K. A low-cost reusable phantom for ultrasound-guided subclavian vein cannulation. Indian J Crit Care Med 2012;16(3):163-165. 
12) Luo B, Yang R, Ying $P$, Awad M, Choti M, Taylor R. Elasticity and Echogenicity Analysis of Agarose Phantoms Mimicking Liver Tumors. Northeast Bioengineering Conference, Easton, PA, 2006. http://www.cs.jhu.edu/ rht/RHT\%20Papers/2006/ Elasticity.pdf, at the date of Nov 7th, 2016.
13) Snedecor GW, Cochran W. Statistical methods. 10th printing. The Iowa State University Press, 1979, Ames, Iowa, USA.

14) Godlee F. How predictive and productive is animal research? BMJ 2014;348:g3719. 